\title{
Photometry with Fabry-Perot Spectrometers
}

\author{
J. Bland-Hawthorn \\ Anglo-Australian Observatory, P.O. Box 296, Epping, NSW 2121, \\ Australia
}

\begin{abstract}
At optical and infrared wavelengths, imaging Fabry-Perot devices are used in three different ways: (i) to obtain a single spectrum of a diffuse source which fills a large fraction of the aperture, (ii) to obtain a monochromatic image within a field defined by the Jacquinot spot, and (iii) to obtain a spectrum at each pixel position over a wide field by tuning the etalon. We review the theoretical basis for Fabry-Perot photometry and summarize the calibration procedures for the different applications. We discuss methods for recognizing and dealing with artefacts (scattered light, atmospheric effects, etc.) which can seriously comprise the photometric integrity of the data if left untreated.
\end{abstract}

\section{Introduction}

Imaging Fabry-Perot interferometers are now in common use at several major observatories and operate at both optical and infrared wavelengths. Traditionally, Fabry-Perots are employed to perform studies of extended gaseous nebulae. Examples include outflow sources (starburst and active galaxies, Herbig-Haro systems) and normal disk galaxies. Some groups have utilised the angular spectral coverage to detect diffuse sources (HI/Ly $\alpha$ clouds in optical emission). More recently, scanning Fabry-Perots have been used to construct spectral line profiles at many pixel positions across a large format CCD. In many instances, these spectra are used simply to obtain kinematic information (line widths, radial velocities) in a strong emission line. It is less common to see these instruments employed as spectrophotometers where the observed signal is calibrated to exoatmospheric flux units. This may be due, in part, to a perception that the nature of the Airy function makes Fabry-Perots unreliable photometers.

In this review, we describe reliable methods for flux-calibrating Fabry-Perot data with the aid of worked examples. We start by comparing the Airy function to other well known functional forms in order to emphasize its distinct properties. This leads to the concept of the 'effective photometric band-pass' which has important implications for the photometric calibration procedure. Accurate photometry does require that the monochromatic and whitelight response of the system are well understood, with the aid of simple numerical simulations if need be. There are many potential pitfalls in the analysis: we illustrate a few of these with data from instruments in Australia, Chile and Hawaii. 


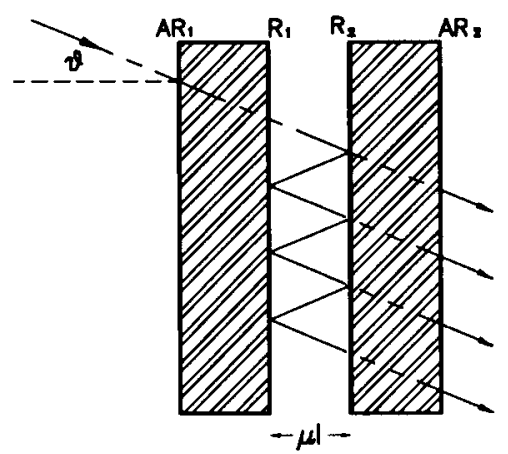

Figure 1. A Fabry-Perot etalon comprising two glass plates with highly reflective interior surfaces, $\mathcal{R}_{1}$ and $\mathcal{R}_{2}$, and anti-reflective exterior coatings, $\mathcal{A R}_{1}$ and $\mathcal{A R}_{2}$.

\section{Airy function}

The most direct route to the Airy function, the instrumental response of the Fabry-Perot, is to use complex exponential notation. In Fig. 1, an incoming plane wave with wavelength $\lambda$ at an angle $\theta$ to the optical axis enters the etalon cavity and performs a series of internal reflections. If the highly reflective inner surfaces have reflectivities of $\mathcal{R}_{1}$ and $\mathcal{R}_{2}$, we can sum over the complex amplitudes of the outgoing plane waves such that

$$
\mathcal{I}=1+\mathcal{R}_{1} \mathcal{R}_{2} e^{2 i \delta}+\mathcal{R}_{1}^{2} \mathcal{R}_{2}^{2} e^{4 i \delta}+\ldots .=\frac{1}{1-\mathcal{R}_{1} \mathcal{R}_{2} e^{2 i \delta}}
$$

in which $2 \delta$ is the phase difference between successive rays. The total transmitted intensity is proportional to the squared modulus of the complex amplitude or

$$
\mathcal{A}=\mathcal{I} \mathcal{I}^{*}=\frac{1}{1+\frac{4 \mathcal{N}^{2}}{\pi^{2}} \sin ^{2} 2 \pi \mu l \lambda^{-1} \cos \theta}
$$

where the refractive index and the plate separation of the cavity are $\mu$ and $l$ respectively, and $\mathcal{N}=\pi \sqrt[1]{\mathcal{R}_{1} \mathcal{R}_{2}}\left(1-\sqrt{\mathcal{R}_{1} \mathcal{R}_{2}}\right)^{-1}$. Clearly, the Airy function has a series of periodic maxima whenever

$$
m \lambda=2 \mu l \cos \theta
$$

which is the well known equation of constructive interference in the $m$ th order.

The quantity $\mathcal{N}$ is called the reflective finesse and depends only on the values of $\mathcal{R}_{1}$ and $\mathcal{R}_{2}$. It is normal procedure to manufacture an etalon with two identical coatings such that $\mathcal{R}_{1}=\mathcal{R}_{2}$. However, in Section 8, we illustrate an important manifestation of a resonating cavity with very different reflection coefficients. 


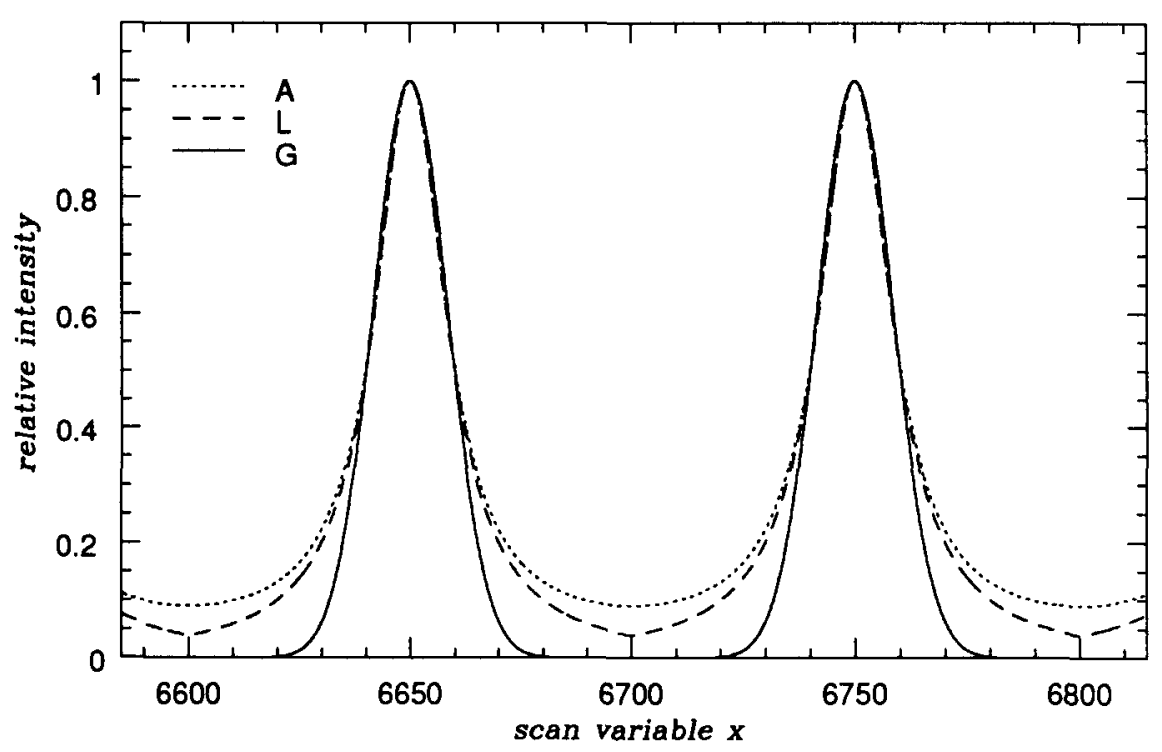

Figure 2. Three cyclic functions listed in Table 1 (column 2) shown at low finesse to emphasize their differences.

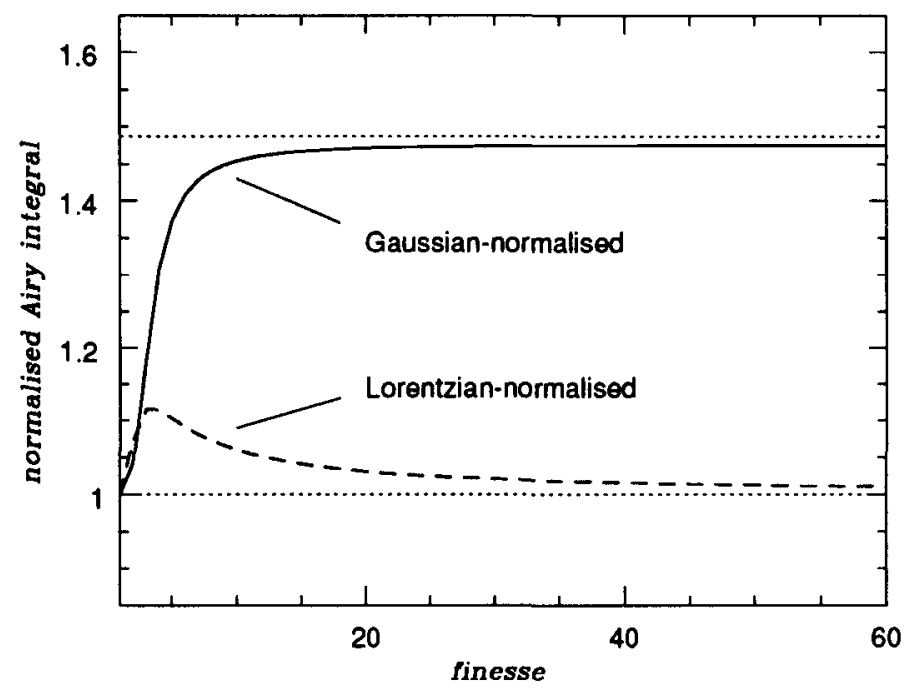

Figure 3. The integral of the Airy curve as a function of finesse normalized with respect to the Gaussian and Lorentzian integrals. The asymptotic limit of the upper curve is $\sqrt{\pi \ln 2}$. 


\begin{tabular}{|l|l|l|}
\hline & $f(x)$ & $\int f(x) d x$ \\
\hline $\mathrm{G}$ & $\exp \left[-\ln 16 \bmod [x, \Delta x]^{2} /(\delta x)^{2}\right]$ & $\left(\frac{\delta x}{2}\right) \sqrt{\frac{\pi}{\ln 2}} \operatorname{erf}\left[\left(\frac{2 \sqrt{\ln 2}}{\delta x}\right) \bmod [x, \Delta x]\right]$ \\
$\mathrm{L}$ & $\left(1+\left(\frac{2}{\delta x}\right)^{2} \bmod [x, \Delta x]^{2}\right)^{-1}$ & $\left(\frac{\delta x}{2}\right) \tan ^{-1}\left[\left(\frac{2}{\delta x}\right) \bmod [x, \Delta x]\right]$ \\
$\mathrm{A}$ & $\left(1+\alpha \sin ^{2}\left(\frac{\pi x}{\Delta x}\right)\right)^{-1}$ & $\left(\frac{\Delta x}{\pi \sqrt{1+\alpha}}\right) \tan ^{-1}\left[\sqrt{1+\alpha} \tan \left(\frac{\pi x}{\Delta x}\right)\right]$ \\
\hline
\end{tabular}

Table 1: Cyclic functions which are periodic over $\Delta x$ with FWHM $\delta x$. The (G)aussian, (L)orentzian and (A)iry functions are illustrated in Fig. 2. The mod function is the modulo function and $\alpha=\left(\frac{2 \Delta_{x}}{\pi \delta}\right)^{2}$. Note that for large $\alpha$, $\left(\frac{\Delta x}{\pi \sqrt{1+\alpha}}\right) \approx\left(\frac{\delta x}{2}\right)$. The gap scanning variable $x$ is offset by $\frac{\Delta x}{2}$ in practice.

In order to arrive at the correct calibration procedure, it is important to understand the nature of the Airy function. Fig. 2 illustrates the three cyclic functions in Table 1 and shows that the area of the Airy function always exceeds the integral of the other functions for a given spectroscopic resolution. By analogy with the Lorentzian profile, the coefficient of the $\sin ^{2}$ factor in equation (2) determines the width of the function. The quantity $\Delta x / \delta x$ is called the effective finesse and $\Delta x$ is the periodic free spectral range.

In Fig. 3, we illustrate how the Airy function, when normalized to the Gaussian and Lorentzian functions, depends on $\mathcal{N}$. In practice, there are factors other than coating reflectivities which contribute to the effective finesse aperture effects, imperfections within the plate coatings, etc. - some of which can serve to make the instrumental response more Gaussian than Lorentzian in form (Atherton et al 1981).

Beyond a finesse of roughly $\mathbf{4 0}$, the Airy function is highly Lorentzian. The reason for this is clear when looking at how the Airy profile has been written in Table 1. At high finesse $(\Delta x>\delta x)$, if we expand $x$ about the peak of the profile, the small angle formula reduces the Airy function to the Lorentzian form. The normalized Airy integral depends only weakly on finesse at large finesse values.

\section{Jacquinot advantage}

There are several approaches to deriving the Jacquinot advantage (Roesler 1974; Thorne 1988), i.e. the throughput advantage of the Fabry-Perot interferometer. By considering the solid angle subtended by the innermost ring and using the small angle formula with equation (3), we arrive at the important relation

$$
\theta^{2}=\frac{2}{R}=\frac{\Omega}{\pi}
$$

which leads to the more familiar form

$$
R \Omega=2 \pi
$$


This equation has been used to demonstrate that Fabry-Perot interferometers, at a given spectroscopic resolution, have a much higher throughput than more conventional techniques (Jacquinot $1954 ; 1960$ ). But the Jacquinot relation has another important consequence. The solid angle of a ring defined by its FWHM intensity points can also be written

$$
\Omega=2 \pi \theta \delta \theta=\pi \lambda(\mathcal{N} \mu l)^{-1}
$$

For a fixed etalon spacing, the solid angle, and hence the spectroscopic resolution, of all rings is a constant. This allows us to write down a simple equation valid for all rings for the signal-to-noise ratio in a monochromatic unresolved line, viz.

$$
\operatorname{SNR}=s(\epsilon \tau \eta)^{0.5}\left(\frac{\delta \lambda^{\prime}}{\delta \lambda}\right)^{0.5}\left(\frac{\Omega}{\omega}\right)^{0.5}\left(s+b+f \delta \lambda\left(\delta \lambda^{\prime} \epsilon \tau\right)^{-1} \sigma_{R}^{2}\right)^{-0.5}
$$

where $s$ and $b$ are the source and background flux (cts pix ${ }^{-1} \mathrm{~s}^{-1}$ ), $\epsilon$ and $\tau$ are the efficiency and exposure times respectively, $\omega$ is the solid angle subtended by a pixeI. The quantity $\delta \lambda^{\prime}$ is the wavelength dispersion $\left(\AA \mathrm{pix}^{-1}\right)$ and $f$ is the number of CCD exposures combined to form the deep spectrum. The factor $\eta$ is discussed below. We normally choose to place the ring center at one corner of the field for two reasons. First, it is always necessary to tilt the etalon in order to throw ghost light out of the field (Section 8). Secondly, the factor $(\Omega / \omega)^{0.5}$ in equation (2) now ensures that the spectroscopic sensitivity is constant over most of the field. There will be an almost linear drop-off in sensitivity at large off-axis angles (far corner from the optical axis) where the rings become seriously incomplete.

An important characteristic of a spectrometer is its 'spectral purity' $\delta \lambda$, i.e. the smallest measurable wavelength difference at a given wavelength (Thorne 1988). This is usually defined as the intensity FWHM of the instrumental profile. When considering the amount of light transmitted by a spectrometer, we need to consider the total area under the instrumental function. This issue is rarely mentioned in the context of long-slit spectrometers partly because their response is highly Gaussian, ${ }^{1}$ in which case the 'effective photometric band-pass' (total area divided by peak height) is very close to the FWHM of the instrumental profile (see Table 1).

At moderate finesse, the Airy function allows through $50 \%$ more light than a Gaussian profile with equal spectroscopic resolution (Fig. 3). Thus, the effective photometric band-pass $\delta \Lambda$ is almost $60 \%$ larger than the band-pass defined by the profile FWHM $\delta \lambda$. The factor $\eta$ in equation (7) corrects for a calculation based on the FWHM of a ring and is defined as $\delta \Lambda / \delta \lambda$. Technically, $\delta \Lambda$ should be adopted as the spectral resolution of the Airy instrumental profile, otherwise we are forced to a serious inconsistency when comparing Fabry-Perot spectrometers to other devices (Bland-Hawthorn \& Jones 1994).

\footnotetext{
${ }^{1}$ The theoretical sinc ${ }^{2}$ response of slit-aperture devices is rarely achieved in practice.
} 


\begin{tabular}{|l|l|l|}
\hline beam & telescope diameter & $3910 \mathrm{~mm}$ \\
& central hole diameter & $1610 \mathrm{~mm}$ \\
& pupil size & $59.9 \mathrm{~mm}$ \\
pupil stop diameter & $45.0 \mathrm{~mm}$ \\
etalon & telescope area & $4.74 \times 10^{6} \mathrm{~mm}^{2}$ \\
& $\begin{array}{l}\text { free spectral range } \\
\text { effective finesse }\end{array}$ & $57.0 \AA$ \\
& $\begin{array}{l}\text { spectral purity } \\
\text { spectral sampling } \\
\text { effective band-pass }\end{array}$ & $1.15 \AA$ \\
& gain & $1.80 \AA$ \\
& read noise & $2.7 \mathrm{e}^{-} / \mathrm{dn}$ \\
& pixel size & $2.3 \mathrm{e}^{-}$ \\
pixel field & $24.0 \mu \mathrm{m}$ \\
\hline
\end{tabular}

Table 2: TAURUS-2 instrumental parameters for a recent observing run at the AAT $3.9 \mathrm{~m}$ using a $50 \mathrm{~mm}$ diameter etalon. The effective telescope area is reduced by the aperture stop.

\section{Photometric calibration procedures}

When we build up a data cube or take a series of observations, it is essential to think of the scan variable as the etalon gap $l$ rather than wavelength. These two variables should never be confused. ${ }^{2}$ The wavelength range is moderated by the filter; the etalon gap is not. The physical plate scanning range is $l_{0} \pm 2 \Delta l$ $=l_{0} \pm 2\left(\lambda_{0} / 2\right)$ where $l_{0}$ is the zeropoint gap and $\Delta l$ is the free spectral range in physical gap units. With this important distinction in mind, for the flux in a standard star observation, we are able to write

$$
S(l)=\int F_{S}(\lambda) \mathcal{A}(\lambda, l) d \lambda
$$

where $F_{S}(\lambda)$ is the product of the stellar spectrum and the filter response. We can write down related expressions for the calibrations. The limits of the integral in equation (9) are defined by the band-pass of the entrance filter. The transform is some form of a convolution equation in that $\mathcal{A}(\lambda, l)$ broadens $F_{S}(\lambda)$ although, technically, the term 'convolution' should be reserved for integrals of the form

$$
S(l)=\int F_{S}(\lambda) \mathcal{A}(\lambda-l) d \lambda
$$

but note that this is a special case of equation (8). Suffice it to say, a spectral line broadened by a spectrometer arises from a convolution and not from a product ${ }^{3}$.

\footnotetext{
${ }^{2}$ The scan axis of a data cube becomes the $\lambda$ dimension only very late in the reduction process.

'This is easy to verify analytically with two Gaussian functions.
} 


\subsection{Narrowband imaging}

The Jacquinot spot is defined as the field about the optical axis within which the peak wavelength variation with field angle does not exceed $\sqrt{2}$ of the etalon bandpass (Jacquinot 1954; Taylor \& Atherton 1980). This angular field can be used to perform close to monochromatic imaging. In this section, we demonstrate how to convert the observed counts to true flux units. We shall assume that the entrance filter selects a band-pass which is a fraction of the free spectral range such that there is no pollution from neighbouring orders.

We determine the TAURUS-2 system efficiency by comparing the observed counts for the flux standard $\eta$ Hyades with the expected counts (Hayes 1970) for which, at $\mathrm{H} \alpha$, we expect $f_{\lambda}=2.65 \times 10^{-11} \mathrm{erg} \mathrm{cm}^{-2} \mathrm{~s}^{-1} \AA^{-1}$ or equivalently $n_{\lambda}=8.69$ phot $\mathrm{cm}^{-2} \mathrm{~s}^{-1} \AA^{-1}$. For the system parameters listed in Table 2, we observed $3.50 \times 10^{4}$ counts for the flux standard in a one second exposure. The efficiency calculation involves corrections for (A) effective photometric band-pass (measured from the calibration data), (B) exposure time, (C) CCD gain, (D) reduced telescope area, and $(E)$ airmass. We now quantify these stages:
A. $3.50 \times 10^{4}$
1.80
$=1.94 \times 10^{4}$ cts $\AA^{-1}$
B.
/ 1.00
$=1.94 \times 10^{4}$
$\operatorname{cts~s^{-1}} \AA^{-1}$
C.
2.70
$=5.25 \times 10^{4}$
elec $\mathrm{s}^{-1} \AA^{-1}$
D.
$4.74 \times 10^{4}=1.11$
E.
1.03
$=1.14$
elec $\mathrm{cm}^{-2} \mathrm{~s}^{-1} \AA^{-1}$
elec $\mathrm{cm}^{-2} \mathrm{~s}^{-1} \AA^{-1}$

It follows that the TAURUS-2 instrumental efficiency is $13.1 \%$ or about $15.0 \pm 0.5 \%$ in the absence of the entrance filter. Thus, we are now able to convert each recorded electron to exoatmospheric flux units.

\subsection{Spectral data cubes}

The important point to realize here is that, with long-slit spectrometers, the instrumental profile is projected onto the detector. In contrast, with scanning Fabry-Perot interferometers, we have the freedom to sample the spectral line of interest however we wish in the scanning dimension. We demonstrate the flux calibration of a spectral scan using the planetary nebula flux standard IC 2165 (Lang 1980). With TAURUS-2, a total of $1.50 \times 10^{6}$ counts was obtained from integrating over the $\mathrm{H} \alpha$ line. The various stages are as before with three additional steps to correct for (F) system efficiency, (G) sampling interval (the same filter was used as before), (H) photon energy at $\mathrm{H} \alpha$.
A. $1.50 \times 10^{6}$
1.80
$=8.33 \times 10^{5}$
cts $\AA^{-1}$
B.
10.0
$=8.33 \times 10^{4}$
cts $\mathbf{s}^{-1} \AA^{-1}$
C.
2.70
$=2.25 \times 10^{5}$
elec s${ }^{-1} \AA^{-1}$
D.
$4.74 \times 10^{4}$
$=4.75$
E.
1.03
$=4.89$
F.
0.131
$=37.3$
G.
* 0.34
$=12.7$
H.
* $3.03 \times 10^{-12}=3.85 \times 10^{-11}$
elec $\mathrm{cm}^{-2} \mathrm{~s}^{-1} \AA^{-1}$ elec $\mathrm{cm}^{-2} \mathrm{~s}^{-1} \AA^{-1}$ phot $\mathrm{cm}^{-2} \mathrm{~s}^{-1} \AA^{-1}$ phot $\mathrm{cm}^{-2} \mathrm{~s}^{-1}$ erg $\mathrm{cm}^{-2} \mathrm{~s}^{-1}$

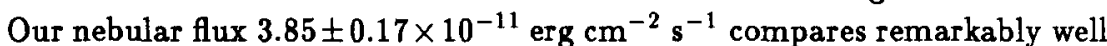
with Lang's value of $3.86 \times 10^{-11} \mathrm{erg} \mathrm{cm}^{-2} \mathrm{~s}^{-1}$ where we have assumed Case B recombination to convert from $\mathrm{H} \beta$ to $\mathrm{H} \alpha$. 

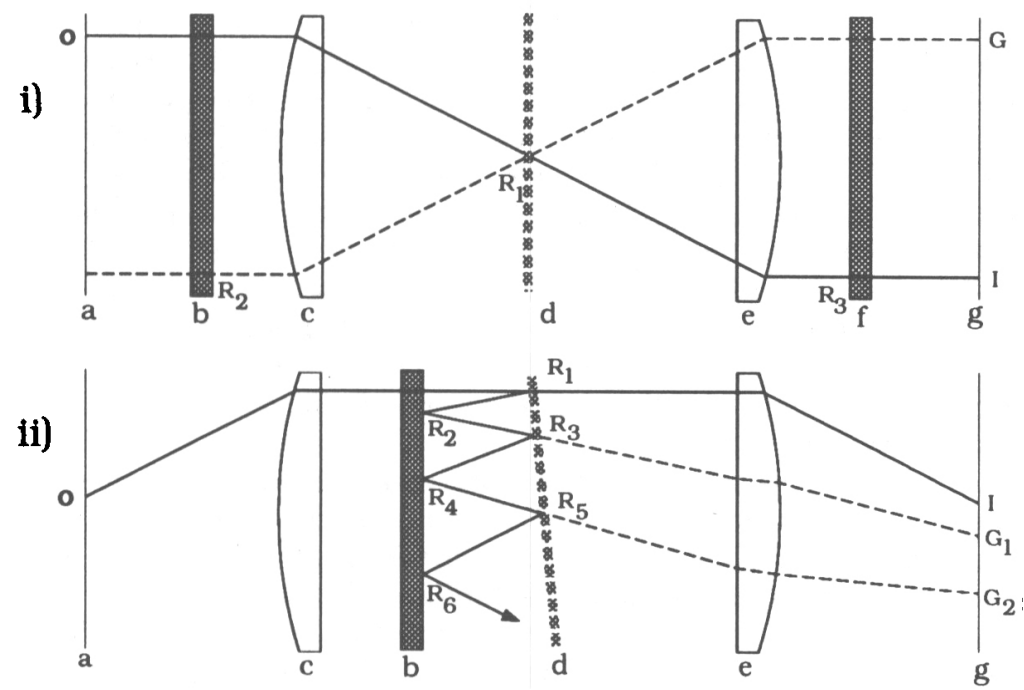

Figure 4. Ghost families arising from internal reflections within a Fabry-Perot spectrometer (see text). (i) Diametric ghosts. Rays from the object $O$ form an inverted image $I$ and an out-of-focus image at $R_{3}$. The reflection at $R_{1}$ produces an out-of-focus image at $R_{2}$. The images at $R_{2}$ and $R_{3}$ appear as a ghost image $G$ at the detector. (ii) Exponential ghosts. The images at $R_{2}$ and $R_{4}$ appear as ghost images $G_{1}$ and $G_{2}$ respectively.

\subsection{Diffuse source detection}

A number of authors have exploited the Jacquinot advantage of the Fabry-Perot to obtain extremely deep spectra of extended, diffuse objects. For a fixed gap spacing, $\lambda \propto \cos \theta$, such that the spectrum in a narrow band is dispersed radially from the optical axis across the field. When the data are binned azimuthally about the optical axis, a single deep spectrum is obtained. Like long-slit spectrometers, the instrumental profile is projected onto the detector but varies across the field according to $\delta \lambda \propto \theta^{-1}$. At the AAT, we have already achieved $\mathrm{H} \alpha$ emission measures of $0.2 \mathrm{~cm}^{-6} \mathrm{pc}\left(2 \times 10^{-19} \mathrm{erg} \mathrm{cm}^{-2} \mathrm{~s}^{-1} \operatorname{arcsec}^{-2}\right)$ at the $3 \sigma$ level in about 90 minutes. In principle, we are able to reach $0.02 \mathrm{~cm}^{-6}$ pc $(3 \sigma)$ in about six hours using 3 " optics. The raw spectrum has quadratic sampling and needs to be resampled to a linear axis where the original number of bins is preserved. As is evident from equation (7), each pixel defines both a spectral interval (applied in step G) and a projected solid angle. We include an additional step (I) to correct for the latter, at which point the final spectrum has units of erg cm $\mathrm{cm}^{-2} \mathrm{~s}^{-1} \AA^{-1} \operatorname{arcsec}^{-2}$. This procedure is to be discussed in more detail elsewhere. 


\section{Whitelight calibration}

It is hard to overstate the importance of the whitelight cube. This is obtained by observing a whitelight source over the same range of etalon spacings used in the actual observations. The whitelight cube maps the response of the filter as a function of position and etalon spacing. There are three effects that we wish to divide out from the data. First, the narrowband filter response, when convolved with the instrumental response, leads to a modulation in the observed spectrum. Secondly, it is well known that filters have variable responses in both collimated and converging beams (Lissberger \& Wilcock 1959). Thirdly, we seek to remove any inhomogeneities in the filter structure as a function of position. Finally, when the whitelight cube is compressed in the spectral dimension, it provides a very high signal-to-noise flatfield for removing pixel-topixel sensitivity variations.

Since it would be impractical to observe a flux standard at every pixel position, we can only flux calibrate the spectral response at each point in the field through the whitelight cube. Thus, we effectively calibrate the whitelight response at the position of the flux standard and thereafter the data cube. It is important to note that a twilight observation provides a better model of uneven illumination across the field. Thus, we replace the low frequency structure in each frame of the whitelight cube with a single high signal-to-noise twilight observation.

\section{Low-level background effects}

Besides the vignette problem, other important low-level effects are CCD fringes and the 'extraneous etalon' Airy pattern (see Section 8). The high-frequency Airy pattern can have a similar amplitude to the CCD fringes. When using narrowband entrance filters, we find that the fringe pattern for some chips can show noticeable variations over a baseline as small as $10 \AA$. This may require co-adding several whitelight cubes to divide out the fringe pattern properly.

\section{Atmospheric attenuation and seeing}

An obvious limitation to good spectrophotometry is the degree of atmospheric stability. The PEPSIOS system made use of a reference channel in order to assess this (Hobbs 1969). With modern day imaging Fabry-Perot systems, we use stars in the field. In good photometric conditions, we find that the stellar intensities, and for that matter, the sky background, map the filter structure rather well. Under these conditions, the CCD always outperforms a photon. counting device (Bland \& Tully 1989). In the presence of cirrus, we often find that the sky continuum follows the filter structure while the stellar response does not, and the stellar FWHM can be highly variable. Such data are of limited use. In certain instances, it may be possible to recover some measure of photometric integrity by spatially filtering the data, particularly in conditions of variable seeing. In the early days, TAURUS used the Image Proportional Photon Counting System to scan rapidly and repeatedly to beat down these 


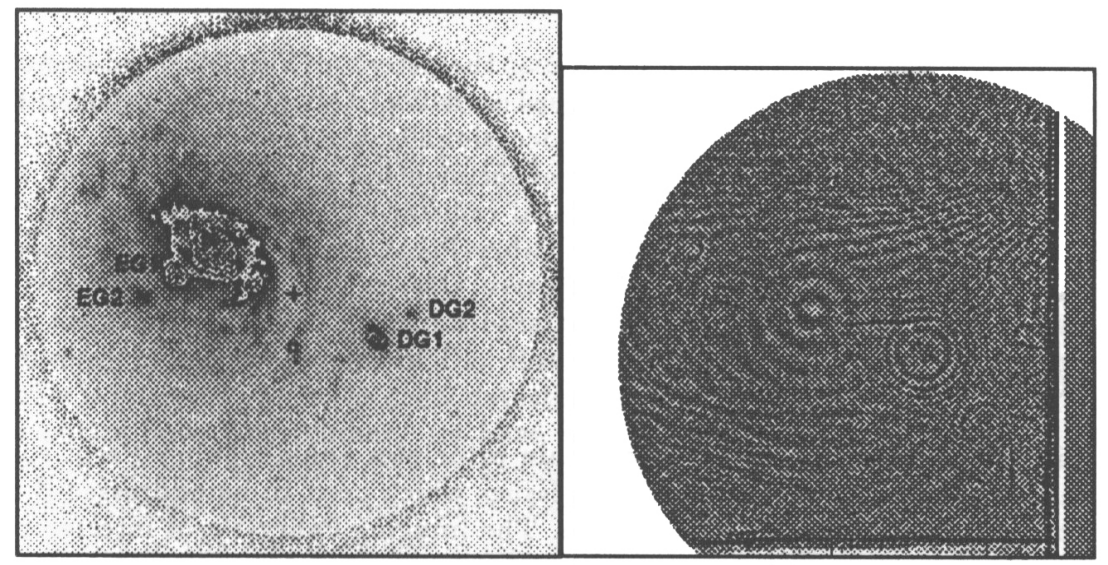

Figure 5. Left.- Two ghost families are seen in this image of NGC 1068 taken with the Rutgers Fabry-Perot on the CTIO $4.0 \mathrm{~m}$ telescope. The optical axis is indicated by the cross. ' $N$ ' is placed slightly to the north of the Seyfert nucleus. 'DG1' is the diametric ghost of the active nucleus; 'EG1' is an exponential ghost of the Seyfert nucleus. 'DG2' is the diametric ghost of 'EG1' and 'EG2' is the exponential ghost of 'EG1'. Right.- The 'extraneous etalon' ghost pattern from the downstream etalon plate (lower right quadrant) and from an air gap in the MOSFP camera (central). The data were taken at the CFHT by illuminating the dome with an H $\alpha$ lamp. The fringe pattern of the Loral \#3 CCD has a similar peak to trough amplitude.

variations (Taylor \& Atherton 1980). To our knowledge, however, these data were never flux calibrated so it is difficult to assess the possible gains.

\section{Ghost families}

Even a minimal Fabry-Perot arrangement can have eight or more optically flat surfaces. At some level, all of these surfaces interact separately to generate spurious reflections. The periodic behavior of the etalon requires that we use a narrowband filter somewhere in the optical path. Typically, the narrowband filter is placed in the converging beam before the collimator or after the camera lens, or in the collimated beam. The filter introduces ghost reflections within the Fabry-Perot optics. The pattern of ghosts imaged at the detector is different in both arrangements, as illustrated in Fig. 4. Examples of these ghosts are shown in Fig. 5. A good way to track these down is to place a regular grid of holes in focus at the focal plane and illuminate the optical system with a whitelight source. We tilt the etalon in such a way that the ghost images of the grid pattern avoid the detector area.

A more difficult problem arises from the optical blanks which form the basis of the etalon. These can act as internally reflecting cavities since, from Section 2 , if we let $\mathcal{R}_{1}=0.96$ and $\mathcal{R}_{2}=\mathcal{A} \mathcal{R}_{1}=0.04$ (air-glass), we see that this generates a ripple pattern with a finesse close to unity. The large optical gap of the outer 
plates produces a high-order Airy pattern at the detector (Fig. 5). Traditionally, the outer surfaces have been wedge-shaped to deflect this spurious signal out of the beam. Even curved lens surfaces occasionally produce 'halation' around point source images which may require experimenting with both bi-convex and plano-convex lenses when designing a focal reducer. At the risk of belabouring the point, it is foolhardy to be using a Fabry-Perot device unless the influence of scattered light is well understood.

\section{Conclusions}

In this review, we have outlined the main principles behind Fabry-Perot spectrophotometry. We have also described issues which need to be addressed if the photometric calibration is to be reliable. The imaging Fabry-Perot interferometer has the capability to provide superior spectrophotometry since slit-aperture devices suffer seeing losses and narrowband filters are tacitly assumed to have constant transmission properties as a function of both position and wavelength. Fabry-Perot interferometers are still not common-user instruments at any observatory for a variety of reasons, most notably because of the restricted wavelength coverage. But for studying extended emission from a few bright lines, the capabilities of the Fabry-Perot are unmatched by any other technique, with the exception of the imaging Fourier Transform spectrometer (e.g., Maillard, this conference). Arguably, the best results have been obtained when Fabry-Perot data are combined with complementary data from narrowband and long-slit devices (see the contributions of Cecil, Hippelein, Pogge, Tully and Veilleux).

It is noteworthy that, in contrast to optical etalons, gap-scanning infrared etalons (e.g. Reay, this conference) have preceded the tremendous on-going advances in infrared detector technology. At optical wavelengths, CCDs continue to improve their performance although there are outstanding areas for future development (e.g., read noise, read-out time). Thus, it would seem that FabryPerot studies, particularly at infrared wavelengths, hold great promise for the future. We only hope that future observing programmes make full use of the spectrophotometric capabilities of the imaging Fabry-Perot interferometer with proper attention to the issues discussed in this review.

Acknowledgments. I would like to thank the Marseilles Observatory for their excellent hospitality. As always, I am indebted to my HIFI colleagues for their inspiration, encouragement and friendship. Gerald Cecil assisted with Figure 4.

\section{Discussion}

Dr. Jockers: Can you explain how you do the whitelight calibration?

J. Bland-Hawthorn: The whitelight calibration is an essential step in the flux calibration. It measures the variation of the filter response (peak transmission, broadening, band-pass shift) as a function of position over the field. To this end, we use the dome flats over a range of etalon gap spacings. We find this approach divides out the wavelength structure fairly well. However, the dome flat illumination does not divide out the vignetting induced by the optical train. 
We sidestep this problem by replacing the low frequency spatial structure within the whitelight cube by a single observation of the twilight sky.

Dr. Boulesteix: Even if we had no idea of the instrumental function, the spectra that you show at the beginning of your talk seemed to be oversampled. Is that true?

J. Bland-Hawthorn: Yes, greatly oversampled in some parts of the spectrum. You are referring to the spectrum obtained from azimuthal binning (or annular summing, in the vernacular of Dr. Roesler). In this instant, the instrumental profile is projected onto the areal detector, analogous to the long-slit case. Once the optics have been chosen, we have no control over this. The heavy oversampling occurs at small off-axis angles. Towards the detector edge, we start to undersample the Airy rings once the quantity $p \lambda \tan \theta$ approaches the spectral purity of the etalon (where $p$ and $\theta$ are the pixel size and off-axis angle in radians).

Dr. Boulesteix: What is the influence of the sampling interval, and what is the best choice for photometry?

J. Bland-Hawthorn: The core of the Airy profile determines the kinematic sampling interval (ideally, half the FWHM of the Airy function). In studies of AGNs, the lines can be so broad that it is possible to increase the sampling interval by at least $50 \%$. The 'effective band-pass' measured from the calibration cube defines the photometric sampling interval which is larger than the kinematic sampling interval.

Dr. Atherton: An imaging Fabry-Perot system designed for high photometric accuracy would have a small field and probably pre-filters of less than half an FSR.

J. Bland-Hawthorn: I agree on both points; the HIFI group tries to ensure the latter condition but the necessary filters are not always at hand.

Dr. Reay: There are two competing ways of minimizing the impact of ghost images formed within the Fabry-Perot plates. Either wedge the plates by $5^{\circ}-10^{\circ}$ to ensure that the ghost is thrown off the detector, or polish the plates optically parallel to ensure that the ghost superimposes exactly on the main image. Such a large wedge angle may have its own problems for the optical designer, but it does ensure that ghosts formed within the plates are eliminated. Polishing the plates optically parallel is easy to do but imposes a high-frequency low amplitude spectral modulation on the signal. Have you considered the trade-offs and, if so, can you comment on your conclusions?

J. Bland-Hawthorn: I have always been of the view that wedging (e.g. Hernandez' text on Fabry-Perot interferometers) is not so difficult to achieve since the outer surfaces do not need to be optically flat. Making the plates optically parallel doesn't help matters. I think the most practical solution is to AR coat the outer surface of the downstream plate so as to beat down the geometric mean of the reflectivities on both surfaces. An overall finesse much less than unity suppresses the amplitude of the fringing. However, the AR coatings with lowest reflectivity $(<0.25 \%)$ tend to have only a narrow band-pass. 


\section{References}

Atherton, P.D., Reay, N.K., Ring, J. \& Hicks, T.R. 1981, Opt. Eng., 20, 806 Bland, J. \& Tully, R.B. 1989, AJ, 98, 723

Bland-Hawthorn, J. \& Jones, A.W. 1994, preprint

Hayes, D.S. 1970, ApJ, 159, 165

Hobbs, L.M. 1969, ApJ, 157, 135

Jacquinot, P. 1954, J. Opt. Soc. Amer., 44, 761

Jacquinot, P. 1960, Rep. Prog. Phys., 23, 267

Lang, K.R. 1980, Astrophysical Formulae, Springer-Verlag, Berlin

Lissberger, P.H. \& Wilcock, W.L. 1959, J. Opt. Soc. Amer., 49, 126

Roesler, F.L. 1974, Meth. Expt. Phys., 12A, chap. 12

Taylor, K. \& Atherton, P.D. 1980, MNRAS, 191, 675

Thorne, A.P. 1988, Spectrophysics, Chapman \& Hall, London 